\title{
Genetic specificity and potential for local adaptation between dengue viruses and mosquito vectors Louis Lambrechts*1,2, Christine Chevillon ${ }^{2}$, Rebecca G Albright ${ }^{1}$, Butsaya Thaisomboonsuk ${ }^{3}$, Jason H Richardson ${ }^{4}$, Richard G Jarman ${ }^{3}$ and Thomas W Scott ${ }^{1}$
}

\begin{abstract}
Address: ${ }^{1}$ Department of Entomology, University of California, One Shields Avenue, Davis, CA 95616, USA, ${ }^{2}$ Génétique et Evolution des Maladies Infectieuses, UMR CNRS-IRD 2724, Centre de Recherche IRD, 911 Avenue Agropolis, B.P. 64501, 34394 Montpellier Cedex 5, France,

${ }^{3}$ Department of Virology, Armed Forces Research Institute of Medical Sciences, 315/6 Rajvithi Road, Bangkok, 10400, Thailand and ${ }^{4}$ Department of Entomology, Armed Forces Research Institute of Medical Sciences, 315/6 Rajvithi Road, Bangkok, 10400, Thailand

Email: Louis Lambrechts* - Louis.Lambrechts@mpl.ird.fr; Christine Chevillon - Christine.Chevillon@mpl.ird.fr; Rebecca G Albright - rgalbright@ucdavis.edu; Butsaya Thaisomboonsuk - ButsayaT@afrims.org; Jason H Richardson - Jason.Richardson@afrims.org; Richard G Jarman - Richard.Jarman@afrims.org; Thomas W Scott - twscott@ucdavis.edu

* Corresponding author
\end{abstract}

Published: 9 July 2009

BMC Evolutionary Biology 2009, 9:160 doi:10.1186/147|-2148-9-160
Received: 17 March 2009

Accepted: 9 July 2009

This article is available from: http://www.biomedcentral.com/147/-2/48/9/160

(c) 2009 Lambrechts et al; licensee BioMed Central Ltd.

This is an Open Access article distributed under the terms of the Creative Commons Attribution License (http://creativecommons.org/licenses/by/2.0), which permits unrestricted use, distribution, and reproduction in any medium, provided the original work is properly cited.

\begin{abstract}
Background: Several observations support the hypothesis that vector-driven selection plays an important role in shaping dengue virus (DENV) genetic diversity. Clustering of DENV genetic diversity at a particular location may reflect underlying genetic structure of vector populations, which combined with specific vector genotype $\times$ virus genotype $(G \times G)$ interactions may promote adaptation of viral lineages to local mosquito vector genotypes. Although spatial structure of vector polymorphism at neutral genetic loci is well-documented, existence of $G \times G$ interactions between mosquito and virus genotypes has not been formally demonstrated in natural populations. Here we measure $G \times G$ interactions in a system representative of a natural situation in Thailand by challenging three isofemale families from field-derived Aedes aegypti with three contemporaneous low-passage isolates of DENV-I.
\end{abstract}

Results: Among indices of vector competence examined, the proportion of mosquitoes with a midgut infection, viral RNA concentration in the body, and quantity of virus disseminated to the head/legs (but not the proportion of infected mosquitoes with a disseminated infection) strongly depended on the specific combinations of isofemale families and viral isolates, demonstrating significant $G \times G$ interactions.

Conclusion: Evidence for genetic specificity of interactions in our simple experimental design indicates that vector competence of Ae. aegypti for DENV is likely governed to a large extent by $G$ $\times G$ interactions in genetically diverse, natural populations. This result challenges the general relevance of conclusions from laboratory systems that consist of a single combination of mosquito and DENV genotypes. Combined with earlier evidence for fine-scale genetic structure of natural Ae. aegypti populations, our finding indicates that the necessary conditions for local DENV adaptation to mosquito vectors are met. 


\section{Background}

Dengue viruses (DENV) are mosquito-borne viruses which, like many RNA viruses, exhibit substantial genetic diversity [1]. This diversity can be hierarchically organized in large clusters of lineages sometimes termed 'genotypes' (the terms lineage and genotype will be used interchangeably hereafter) within each one of the four distinct serotypes (DENV-1, -2, -3 and -4) [2]. In the last 200 years, the number of DENV lineages worldwide has been increasing exponentially [3], along with increasing epidemic frequency and occurrence of severe forms of the disease $[4,5]$. Dengue is now the most common human arthropod-borne viral (arboviral) disease and a major public health threat [6]. Although DENV genetic variation alone is not sufficient to completely explain the incidence of severe disease or the magnitude of outbreaks, there is compelling evidence for differences in virulence and epidemic potential among DENV lineages (reviewed in [2]). Understanding the evolutionary processes shaping the increasing diversity of DENV lineages will, therefore, provide important insights into the mechanisms regulating epidemics and pathogenicity associated with genetic variation among viruses [1].

The spatial distribution of DENV genetic diversity generally reveals a population structure whereby geographic subdivisions reflect greater gene flow within than between subdivisions (reviewed in [7]). Results from a recent study indicated that such a genetic structure can be observed on a fine spatial grid [8]; phylogenetic differences were detected among DENV isolates recovered from schools separated by only a few kilometers in rural Thailand. Although multiple DENV lineages co-circulated within individual schools, there was strong genetic differentiation among lineages between schools that remained stable over the 10-month sampling period [8]. Thus, despite frequent viral migration into the area, individual schools located a few kilometers apart represented distinct DENV evolutionary entities. A fundamental unanswered question concerns the relative influence of natural selection (adaptive evolution) and genetic drift (neutral evolution) on the genetic structure of DENV populations [9]. Although it is clear that stochastic processes play a significant role in shaping DENV genetic diversity $[10,11]$, genetic signatures of adaptive evolution have been recurrently detected in natural DENV isolates [12-16].

Among evolutionary forces actively driving the evolution of arboviruses, vector-driven selection may play an important role by selecting lineages that are better suited for mosquito transmission [9]. For instance, the emergence of chikungunya virus (CHIKV) in the Indian Ocean in 2004, and subsequent spread to India and Europe was attributed to the acquisition of a single adaptive mutation that enhances transmission efficiency by Aedes albopictus $[17,18]$. This mutation confers a selective advantage in locations where Ae. albopictus predominates over Ae. aegypti, which is usually considered the primary vector of CHIKV. Similarly, the emergence of a new lineage of West Nile virus in North America was attributed to earlier and more efficient transmission by Culex mosquitoes relative to the lineage that initially colonized this part of the world [19]. DENV are no exception in this regard. Displacement of the American (AM) DENV-2 genotype by a Southeast Asian (SA) DENV-2 genotype in the Western Hemisphere was associated with more efficient infection and dissemination in Ae. aegypti of the SA than AM genotype [20-22]. Likewise, an invasive DENV-3 isolate from Sri Lanka infected and disseminated more efficiently in Ae. aegypti mosquitoes than a displaced, native DENV-3 isolate [23]. Interestingly, in both DENV examples above, outcompeted genotypes tended to cause mild disease whereas invasive genotypes correlated with more severe disease manifestations. This supports the view that enhanced vector transmission, among other factors, may contribute to the evolutionary success of lineages that are more virulent to humans [2].

The potential role of vector-driven selection in DENV evolution raises the question whether the genetic structure of DENV populations reflects, at least partly, that of their vectors. Indeed, Ae. aegypti distribution consists of a patchwork of genetically differentiated populations [2427]. Because the vector competence of Ae. aegypti for DENV is in part genetically determined (reviewed in [28]), it has been hypothesized that the structure of DENV populations may partly result from the adaptation of viruses to the local vector genotypes [9]. Under this hypothesis, DENV transmission would be more efficient by local vector genotypes (sympatric vector-virus pairs) than by vector genotypes from distant populations (allopatric vector-virus pairs). Pathogen adaptation to local hosts has been reported in a variety of systems (reviewed in [29]), including malaria parasites and their mosquito vectors [30]. Evolution of local adaptation has been predicted when the pathogen has an evolutionary advantage over the host, such as higher mutation rate, shorter generation time, higher migration rate, and larger population size [31-33]. These conditions could undoubtedly apply to the DENV-Ae. aegypti system [9].

An additional necessary condition for the occurrence of DENV local adaptation to Ae. aegypti is that the genetic structure of mosquito populations must be coupled with some degree of genetic specificity of vector-virus compatibility. In other words, DENV transmission by Ae. aegypti must be determined, at least partly, by genotype $\times$ genotype $(G \times G)$ interactions. Such $G \times G$ interactions, whereby the infection outcome depends on the specific combination of host and pathogen genotypes, are found in many systems [34]. The effects of vector and virus genotypes, independently, on DENV transmission by $A e$. 
aegypti are well-documented. Variation in vector competence for a reference DENV isolate among different geographic strains of $A e$. aegypti has been repeatedly reported [35-38], and can often be directly correlated to genetic differences $[39,40]$. Reciprocally, DENV isolates have been shown to vary in their infectivity to a given mosquito strain $[20,22]$. Results from an earlier study showing some degree of interaction specificity between laboratory strains of mosquitoes and DENV serotypes and isolates [41] and from another study where SA genotypes of DENV-2 performed better than AM genotypes in two field-derived populations of Ae. aegypti, but not in a laboratory adapted colony [21] were suggestive of genetic specificity of vectorvirus compatibility. However, the occurrence of $\mathrm{G} \times \mathrm{G}$ interactions between DENV and Ae. aegypti has not been formally quantified in natural populations.

Here, we measured the extent of $G \times G$ interactions between DENV and mosquito genotypes that are naturally interacting in the field. We challenged three Ae. aegypti isofemale families derived from a wild population sampled in 2007 in Ratchaburi, Thailand with three low-passage DENV-1 viruses that were isolated the same year from humans (in Bangkok, Kamphaeng Phet, and Ratchaburi, respectively). Following standard methods of quantitative genetics [34], we estimated the extent to which the outcome of the infection is determined by $\mathrm{G} \times \mathrm{G}$ interactions by measuring the statistical effect of isofemale family $\times$ virus isolate interactions on several indices of vector competence, defined as the intrinsic permissiveness of a vector to become infected and subsequently transmit a pathogen [42].

\section{Results}

Overall, vector competence was scored in 333 female Ae. aegypti. Each combination of isofemale family and virus isolate was represented by 28-53 individuals (mean $=37$, median $=36$ ) divided into two experimental blocks of $11-28$ individuals $($ mean $=18.5$, median $=19)$. The three DENV-1 isolates used in this study were collected within a one-month period at three locations in Thailand (Bangkok: BKK, Kamphaeng Phet: KPP, and Ratchaburi: RTB) and had identical passage histories (Table 1). Estimated virus titers in the blood meals were remarkably similar across isolates and experimental blocks, with all titers ranging within a third of a log unit per ml (Table 1 ). This allowed us to assume that most of the phenotypic differences observed between isolates were due to genetic differences. Across families and isolates, 63.1\% of females were infected, and $42.9 \%$ had a disseminated infection. Isofemale families differed significantly in wing length (S.S. $\left.=0.052, F_{2,57}=3.85, P=0.027\right)$, indicating significant genetic variation in body size. The mean wing length $( \pm \mathrm{SE})$ was $3.11 \pm 0.018,3.08 \pm 0.018$, and $3.04 \pm 0.018$ $\mathrm{mm}$ for families $\mathrm{A}, \mathrm{B}$, and $\mathrm{C}$, respectively. Corresponding mean age at pupation $( \pm$ SE) was $7.29 \pm 0.035,7.15 \pm$ 0.037 , and $7.28 \pm 0.033$ days, respectively. Although three data points are not enough to obtain a valid correlation, it is worth noting that the overall proportion of infected mosquitoes by family tended to be negatively correlated with their mean wing length (linear regression: $\mathrm{R}^{2}=0.97$, $P=0.102)$, supporting the view that larger females are more resistant to dengue infection than small females [43].

Two factors had a significant influence on end-point mortality of adult mosquitoes at 14 days post-infection (dpi). Mortality significantly differed between blocks (S.S. = $\left.0.245, F_{1,4}=19.5, P=0.012\right)$, and marginally significantly between virus isolates (S.S. $=0.178, F_{2,4}=7.08, P=0.049$ ). Although $5.8 \%$ of females in the first block died before 14 dpi, $16.3 \%$ of females in the second block did so, possibly due to the one-day age difference. Across blocks, endpoint mortality was $5.3 \%, 11.1 \%$, and $16.1 \%$ for the KPP, $\mathrm{BKK}$, and RTB isolates, respectively. Mortality did not depend on the family-isolate combinations (S.S. $=0.131$, $\left.F_{4,4}=2.62, P=0.19\right)$.

The proportion of infected mosquitoes strongly depended on the family, the isolate, and most importantly in the context of this study, their interaction (Table 2a; Figure 1a). The percentage of infected mosquitoes ranged from $30.2 \%$ to $100 \%$ among family-isolate combinations. The interaction effect appeared to be mainly due to the combination of the RTB isolate and mosquito family $\mathrm{B}$ for which the proportion of infected mosquitoes was lower

Table I: Description of DENV-I isolates used in this study

\begin{tabular}{llllll}
\hline Isolate & Date collected & Location & Passage history & $\begin{array}{l}\text { Blood meal titer, block I } \\
\text { (FFUs/mI) }\end{array}$ & $\begin{array}{l}\text { Blood meal titer, block 2 } \\
\text { (FFUs/ml) }\end{array}$ \\
\hline BKK & 27 July 2007 & Bangkok & C6/36-5 & $5.5 \times 10^{6}$ & $4.0 \times 10^{6}$ \\
\hline RTB & 24 July 2007 & Ratchaburi & C6/36-5 & $5.0 \times 10^{6}$ & $3.4 \times 10^{6}$ \\
\hline KPP & 19 Aug 2007 & Kamphaeng Phet & C6/36-5 & $2.9 \times 10^{6}$ & $2.0 \times 10^{6}$
\end{tabular}

For each isolate, the date and location of collection, passage history, and blood meal titers for both experimental blocks are indicated. Blood meal titers were estimated by fluorescent focus assay (FFA) in C6/36 cells. 
Table 2: Test statistics of categorical vector competence indices

\begin{tabular}{llllll}
\hline & \multicolumn{4}{l}{ (a) Proportion infected } & (b) Proportion infected with disseminated infection \\
\hline Source of variation & d.f. & L-R $\chi^{2}$ & P-value & L-R $\chi^{2}$ & $P$-value \\
\hline Family & 2 & 16.2 & 0.0003 & 2.34 & 0.3107 \\
\hline Isolate & 2 & 29.4 & $<0.0001$ & 38.8 & 0.0001 \\
\hline Family*Isolate & 4 & 14.8 & 0.0051 & 6.28 & 0.1793 \\
\hline Block & 1 & 0 & 1.0 & 0 & 0.9882 \\
\hline Family*Block & 2 & 0.64 & 0.7247 & 0.84 & 0.6574 \\
\hline Isolate*Block & 2 & 0.64 & 0.7262 & 0.27 & 0.8753 \\
\hline Family*Isolate*Block & 4 & 1.29 & 0.8634 & 11.9 & 0.0183
\end{tabular}

The table shows the nominal logistic regression for the proportion of (a) mosquitoes with detectable viral RNA in their bodies (thorax+abdomen) and (b) infected mosquitoes (excluding uninfected) with a disseminated infection in their head/legs (determined by FFA in Vero cells) as a function of mosquito isofemale families, virus isolates, experimental blocks, and their interactions.

than would have been expected from examination of the other combinations (Figure 1a). Because the family $\times$ isolate $\times$ block three-way interaction was not statistically significant, our results indicated that the effect of the family $x$ isolate interaction was consistent across blocks (Table $2 a)$. In other words, small differences in mosquito age or blood meal titer between blocks did not affect the probability of infection in a given mosquito-virus combination.
In contrast, the proportion of mosquitoes with a disseminated infection among those successfully infected was only significantly influenced by the virus isolate (Table 1b). More than $90 \%$ mosquitoes infected by the RTB isolate had a disseminated infection whereas this percentage ranged from $40 \%$ to $70 \%$ for the BKK and KPP isolates (Figure 1b). Despite some degree of interaction indicated by crossing lines (Figure $1 \mathrm{~b}$ ), the family $\times$ isolate interac-
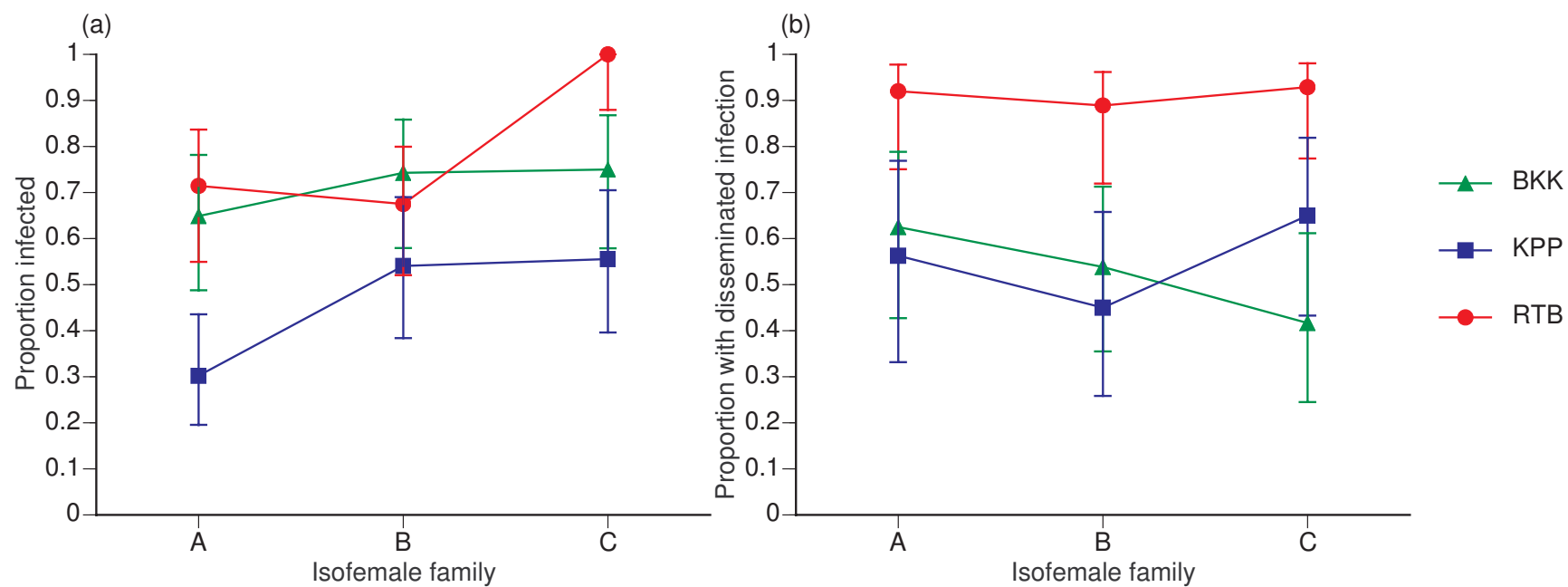

Figure I

Effect of family $\times$ isolate interactions on virus infection and dissemination. (a) The proportion of mosquitoes with a midgut infection and (b) proportion of infected mosquitoes with a disseminated infection as a function of mosquito families and virus isolates. In both panels, three isofemale families from a Ratchaburi population (A, B, and C) are ranked on the $x$-axis according to the mean proportion of infected mosquitoes across isolates. Each line represents a single virus isolate (BKK: Bangkok; KPP: Kamphaeng Phet; RTB: Ratchaburi). Vertical bars show the confidence intervals of the proportions. Crossing lines give an indication of family $\times$ isolate interactions. 
tion was not statistically significant, possibly due to variation between experimental blocks as indicated by the significant family $\times$ isolate $\times$ block three-way interaction (Table 1b). Moreover, exclusion of the 123 uninfected mosquitoes reduced statistical power of this analysis.

Viral RNA concentrations in the body of infected mosquitoes and the number of fluorescent focus units (FFUs) in the head/legs of mosquitoes with a disseminated infection varied substantially among family-isolate combinations, with varying ranking orders of viral isolates for each isofemale family (Figure 2 ). Both variables were significantly influenced by the family $\times$ isolate interaction, with no significant difference between experimental blocks (Table 3). Regardless of their ranking order in a given mosquito family, the general profile of each isolate shared some degree of similarity between viral RNA concentration and numbers of disseminated FFUs (Figure 2), possibly reflecting differing viral growth capacities in different mosquito genetic backgrounds. Viral RNA concentration in infected mosquitoes did not appear to be correlated to the proportion of infected mosquitoes or the proportion of mosquitoes with a disseminated infection, indicating that the probability of these events may be largely independent of the efficiency of viral genome replication.

\section{Discussion}

Results of our experiment strongly support the hypothesis that vector competence of Ae. aegypti for DENV-1 is governed by $\mathrm{G} \times \mathrm{G}$ interactions. With the exception of virus dissemination success, all of the indices of vector compe- tence we examined (midgut infection success, viral RNA concentration in bodies, virus titer in head/legs) were at least partly determined by the specific combination of mosquito family and virus isolate. The use of a randomized complete block design allowed us to rule out the possibility of environmental bias by confirming the consistency of family $\times$ isolate interactions effect across experimental blocks.

Our conclusions are based on the premise that family $x$ isolate interactions reliably approximate $\mathrm{G} \times \mathrm{G}$ interactions [34]; i.e., in our design observed phenotypic differences truly reflect the underlying genetic differences between mosquitoes and viruses. The use of isofemale families to assess the genetic basis of a trait assumes that the ratio of among- to within-families variations is proportionate to the heritable variation for that trait [44]. This assumption is reasonable as long as environmental variation in rearing conditions or parental effects do not differ between families. We ensured homogeneity by maintaining the $\mathrm{F}_{0}-\mathrm{F}_{2}$ parental generations under standard laboratory conditions and rearing the $\mathrm{F}_{3}$ individuals under strictly identical conditions. Non-genetic differences in virus infectivity were minimized by growing the viruses simultaneously under the same conditions and exposing mosquitoes to very similar blood meal titers (Table 1). Uncontrolled, slight differences in blood meal titers did not appear to influence results. For instance, the RTB isolate produced the highest average values across families for all vector competence indices, although the BKK isolate had the highest blood meal titers in both

Table 3: Test statistics of continuous vector competence indices

\begin{tabular}{lllllllll}
\hline & \multicolumn{7}{l}{ (a) Viral RNA concentration in body } & (b) Mean virus titer in head/legs \\
\hline Source of variation & d.f. & S.S. & $\boldsymbol{F}$ & $\boldsymbol{P}$-value & d.f. & S.S. & $\boldsymbol{F}$ & $\boldsymbol{P}$-value \\
\hline Family & 2 & 0.63 & 2.01 & 0.1374 & 2 & 1.53 & 2.22 & 0.1127 \\
\hline Isolate & 2 & 0.59 & 1.88 & 0.1550 & 2 & 1.45 & 2.10 & 0.1266 \\
\hline Family*lsolate & 4 & 2.09 & 3.33 & 0.0115 & 4 & 3.58 & 2.60 & 0.0394 \\
\hline Block & 1 & 0.02 & 0.14 & 0.7126 & 1 & 0.83 & 2.42 & 0.1223 \\
\hline Family*Block & 2 & 0.01 & 0.03 & 0.9668 & 2 & 0.44 & 0.63 & 0.5328 \\
\hline Isolate*Block & 2 & 0.29 & 0.93 & 0.3949 & 2 & 0.83 & 1.20 & 0.3053 \\
\hline Family*Isolate*Block & 4 & 0.87 & 1.38 & 0.2417 & 4 & 1.27 & 0.92 & 0.4530 \\
\hline Error & 192 & 30.1 & & & 125 & 43.1 & \\
\hline
\end{tabular}

Results from analysis of variance of (a) viral RNA concentration in the bodies (thorax+abdomen) of infected mosquitoes and (b) mean virus titer (determined by FFA in Vero cells) in the head/legs of mosquitoes with a disseminated infection as a function of mosquito isofemale families, virus isolates, experimental blocks, and their interactions. 

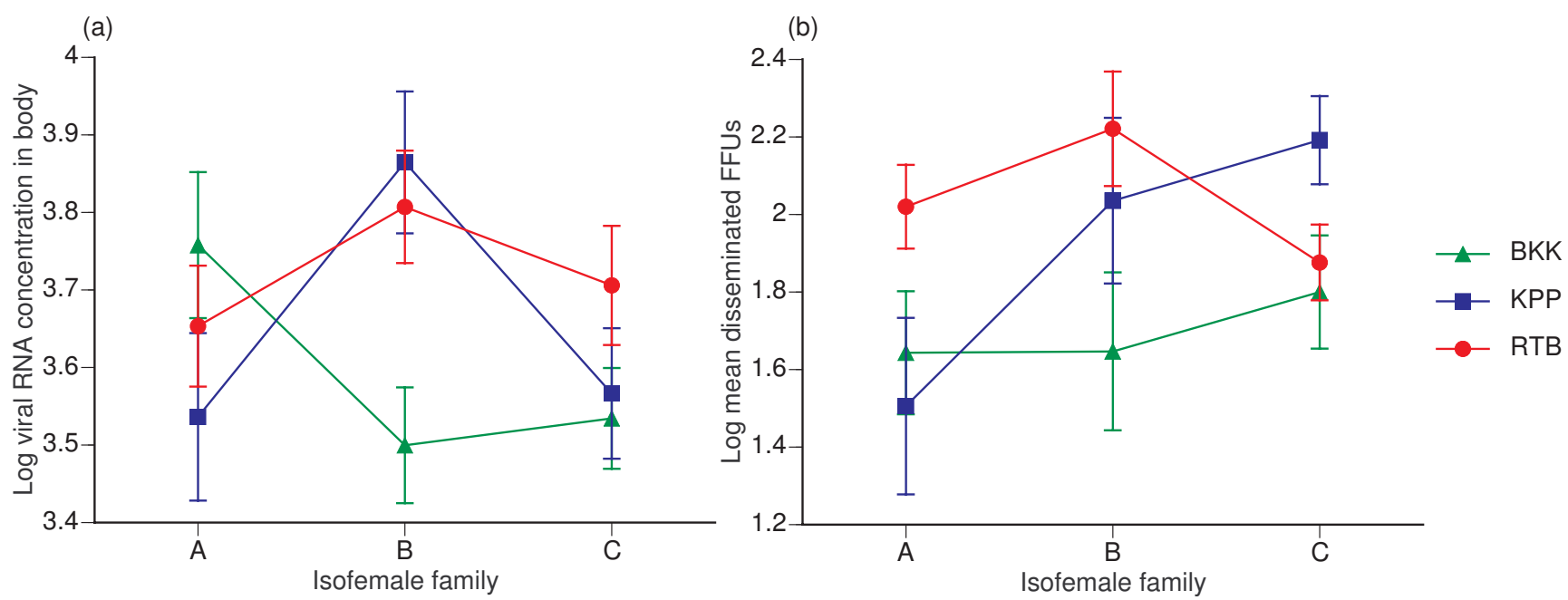

\section{Figure 2}

Effect of family $x$ isolate interactions on viral RNA concentration in mosquito bodies and virus titer in heads/ legs. (a) The log-transformed viral RNA copy number per $\mu$ l of homogenized body (thorax+abdomen) in infected mosquitoes and (b) log-transformed mean number of fluorescent focus units (FFUs) in the head/legs of mosquitoes with a disseminated infection as a function of mosquito families and virus isolates. In both panels, three isofemale families from a Ratchaburi population $(A, B$, and $C$ ) are ranked on the $x$-axis according to the mean proportion of infected mosquitoes across isolates (consistently with Figure I). Each line represents a single virus isolate (BKK: Bangkok; KPP: Kamphaeng Phet; RTB: Ratchaburi). Each point represents the mean and vertical bars are the standard errors of the means. Crossing lines give an indication of family $x$ isolate interactions.

experimental blocks. Although virus amplification in cell culture may have imposed a selective pressure on the original viruses, the low number of passages allowed us to assume that we still had isolates representative of wildtype viral genetic diversity. Overall, it seemed reasonable to conclude that our approach provided a reliable estimate of $G \times G$ interactions in this system.

It is remarkable that we were able to detect $\mathrm{G} \times \mathrm{G}$ interactions using a very restricted experimental design (three mosquito families $\times$ three virus isolates). Observing a highly significant effect of the interaction term with limited statistical power indicates that $G \times G$ interactions likely represent a strong determinant of vector competence in natural Ae. aegypti populations, which display a much higher level of genetic polymorphism than was captured by our three isofemale families sampled at a single location. We expect $G \times G$ interactions to be even more prominent when mosquito genotypes from more genetically distinct populations are examined. Indeed, a nested analysis of Ae. aegypti mitochondrial haplotype frequencies in Thailand showed that while $57 \%$ of the total variation was found within collections, $18 \%$ and $25 \%$ of that variation were found among samples collected within a distance of $25 \mathrm{~km}$ and more than $100 \mathrm{~km}$ apart, respectively [25]. Moreover, because the three DENV-1 isolates used in this study were all collected in Thailand in 2007, it is likely that they are closely genetically related. We expect that $\mathrm{G} \times \mathrm{G}$ interactions will occur to a greater extent when the analysis includes more distantly related viral lineages or even different serotypes.

A practical implication of our results is that the conclusions drawn from one particular vector-virus combination (such as one mosquito population challenged with a reference DENV isolate) are not likely to be representative of other combinations. This challenges the broad-scale relevance of genetic loci associated with vector competence that were identified in laboratory-tractable systems. A recent meta-analysis showed that quantitative trait loci (QTL) controlling host resistance against one pathogen isolate were on average recovered in only $24 \%$ of the cases where infections with different isolates were investigated; i.e., each particular host-pathogen combination was based on a different set of QTL and epistatic interactions [45]. G $\times$ G interactions may help to explain why the QTL controlling midgut infection by a Jamaican DENV-2 isolate in highly selected lines of Ae. aegypti $[46,47]$ did not correlate with those previously identified in field-derived Ae. aegypti challenged with a Puerto Rican DENV-2 isolate [48]. Likewise, it sheds a new light on a study where DENV-2 isolates of the SA genotype performed better than isolates of the AM genotype in two field-derived Ae. aegypti populations, but not in a laboratory-adapted colony [21]. 
Although additional studies are warranted to confirm the impact of $\mathrm{G} \times \mathrm{G}$ interactions on actual virus transmission through an infectious bite, their occurrence during DENV incubation in Ae. aegypti provides important insights into the mechanisms shaping DENV population structure and evolution. It helps to explain why despite strong genetic bottlenecks during mosquito transmission, major DENV variants are generally conserved in both vertebrate and arthropod hosts $[49,50]$. Indeed, $G \times \mathrm{G}$ interactions indicate that elimination of viruses that are incompatible with local mosquito genotypes may counteract the effect of genetic drift in the form of purifying selection at the population level. Likewise, the observed spatial clustering of DENV lineages on a restricted spatial scale [8] may in fact be promoted by location-specific vector-driven selection. Over time, genetic specificity of vector-virus compatibility would combine with the genetic structure of Ae. aegypti populations to drive the adaptation of DENV to increased compatibility with local mosquito genotypes [9]. Interestingly, it is worth noting that in our experiment, while the three isofemale vector families were derived from samples collected in Ratchaburi, all of the vector competence indices were highest, on average, for the viral isolate from that same location (RTB). Although this does not constitute firm evidence for local adaptation of DENV, it is consistent with the hypothesis that DENV transmission may be achieved with greater success by sympatric than by allopatric vector-virus pairs. Inclusion of mosquito families from different populations in a similar design will help to more definitively address this question. Future research using multiple replicates of sympatric and allopatric pairs will determine the extent of local adaptation in this system. It is clear that factors other than vector-mediated population structure contribute to the evolution of certain DENV lineages; i.e., 'cosmopolitan' genotypes that are found across broad geographical distributions. We speculate, however, that to some extent the spatial organization of DENV populations may reflect the geographical distribution of Ae. aegypti genotypes, which would be similar to what was demonstrated (at the species level) for Mexican populations of Plasmodium vivax and their Anopheles vectors [30].

\section{Conclusion}

We demonstrate that the vector competence of Ae. aegypti for DENV-1 is determined by $\mathrm{G} \times \mathrm{G}$ interactions in which potential for mosquito infection and virus transmission depends on the specific combination of vector and virus genotypes. This observation challenges the general relevance of genetic loci controlling vector competence that were identified using a single combination of mosquito and virus genotypes. The combination of $G \times G$ interactions and spatial genetic structure of vector populations is consistent with the potential for DENV adaptation to local vectors. Mosquito vector-driven selection may play a more important role in DENV microevolution than previously thought.

\section{Methods General design}

Because we wanted to (i) use a representative sample of a natural mosquito population and (ii) minimize parental effects due to the variability in environmental conditions [51], we used $\mathrm{F}_{3}$ isofemale families of Ae. aegypti generated from field-sampled immatures (larvae and pupae) raised for two generations in the laboratory. Isofemale families consist of the progeny of individual females. They are classically used in studies of quantitative genetics to investigate the genetic basis of a trait (e.g., [52]). Although Ae. aegypti females can mate multiple times, they are thought to be monandrous due to male accessory gland proteins that are transferred during copulation and induce subsequent sexual refractoriness [53]. Based on the assumption that sibs are genetically more similar than non-sibs, one can estimate the extent to which a trait has a genetic basis by comparing the phenotypic variations observed within and between families [44]. Using this approach genetic effects are confounded with potential maternal effects, which can be controlled by rearing the parental generations under standard homogenous conditions. Our experiment was based on a reciprocal cross-infection design involving three Ae. aegypti isofemale families and three DENV-1 isolates. The experiment was duplicated in two separate experimental blocks by splitting $\mathrm{F}_{3}$ mosquitoes from the same batch into two groups and infecting each group on two successive days with the same viral culture harvested one day apart. This procedure allowed us to assess the repeatability of results while controlling for nuisance factors introduced by duplication of the experiment (e.g., mosquito age, virus titer). We measured $\mathrm{G} \times \mathrm{G}$ interactions with the statistical effect of the family $\times$ isolate two-way interaction on vector competence [34]. We estimated the repeatability of the results with the statistical significance of the family $\times$ isolate $\times$ block three-way interaction as an indication of the degree of consistency of the two-way interaction effect across blocks.

\section{Mosquitoes}

The $F_{0}$ generation was initiated with a large number $(>1,500)$ of immatures collected from multiple (3-6) artificial containers in each of 10-12 households in Don Thako, Muang district, Ratchaburi (Thailand) during September 2007. Adults were allowed to emerge in the laboratory, mate randomly, and feed on anesthetized hamsters as a blood source. Eggs were collected and stored on dry pieces of paper towel maintained under high humidity. Mosquitoes in $\mathrm{F}_{1}-\mathrm{F}_{3}$ generations were raised under standard insectary conditions at $27 \pm 1^{\circ} \mathrm{C}$, high humidity, and under 12:12 hour light:dark cycle. Eggs were hatched synchronously by placing them under low pressure for 30 
min and larvae were reared in $25 \times 40 \mathrm{~cm}$ plastic trays filled with $1.5 \mathrm{l}$ of deionized water at a density of approximately 200 individuals per tray. $\mathrm{F}_{1}-\mathrm{F}_{2}$ larvae were fed on a 1:1 mix of ground puppy chow and bovine liver powder ( $0.05 \mathrm{~g}$ /tray on days 0,1 , and $2 ; 0.1 \mathrm{~g} /$ tray on day $3 ; 0.2 \mathrm{~g} /$ tray on day $4 ; 0.3 \mathrm{~g} /$ tray on day 5 ; and $0.2 /$ tray on days 6 and 7). Adults were housed in large cages with permanent access to $10 \%$ sucrose. $\mathrm{F}_{1}$ females were fed on defibrinated sheep blood (Quad Five, Ryegate, MT) through pieces of desalted porcine intestine stretched over water-jacketed glass feeders. $\mathrm{F}_{2}$ females were allowed to mate randomly for three days and then housed individually. They were daily offered a blood meal on a human arm and allowed to lay eggs on wet filter papers. In order to obtain a large number of $\mathrm{F}_{3}$ females in each family, egg batches from multiple (4-6) gonotrophic cycles were combined and hatched simultaneously. $\mathrm{F}_{3}$ larvae were reared at a low density (approximately 125 per tray) to maximize their survival. Larvae of each family were reared in several trays that were moved daily at random between the shelves of the insectary to minimize any uncontrolled environmental variation. They were fed on a $1: 1 \mathrm{mix}$ of ground puppy chow and bovine liver powder $(0.02 \mathrm{~g} /$ tray on day $0 ; 0.04$ $\mathrm{g} /$ tray on day $1 ; 0.06 \mathrm{~g} /$ tray on day $2 ; 0.08 \mathrm{~g} /$ tray on day $3 ;$ $0.1 \mathrm{~g} /$ tray on day $4 ; 0.2 \mathrm{~g} /$ tray on day 5 ; and $0.1 /$ tray on days 6 and 7). Female body size was estimated for each $F_{3}$ isofemale family by measuring the wing lengths of a random sample of 20 individuals per family. Wings were measured from the tip (excluding the fringe) to the distal end of the alula with a precision of $\pm 0.01 \mathrm{~mm}$ using a dissecting microscope equipped with a micrometer. All experimental infections were conducted using $\mathrm{F}_{3}$ females.

\section{Infection}

We used three DENV-1 isolates that were recovered during July-August 2007 as part of routine surveillance diagnostic procedures at the Armed Forces Research Institute of Medical Sciences (AFRIMS) Bangkok laboratory from the serum of clinically ill dengue patients attending hospitals in Bangkok, Ratchaburi, and Kamphaeng Phet. Each diagnostic isolate underwent a strictly identical passage history (Table 1). Five passages in cell culture was the minimum number required to obtain a titer sufficiently high to infect mosquitoes orally using an artificial blood meal. Confluent cultures of Ae. albopictus cells (C6/36, ATCC \#CRL-1660) in 6-well plates were inoculated with virus at a multiplicity of infection of 0.01 and incubated at $28^{\circ} \mathrm{C}$ under $5 \% \mathrm{CO}_{2}$. Half of the supernatant was replaced with fresh medium $7 \mathrm{dpi}$. Cells and medium were harvested at 11 and 12 dpi to prepare the infectious blood meal of experimental blocks 1 and 2, respectively. The blood meal consisted of 1:1 mix of defibrinated sheep blood (Quad Five) and virus suspension. Three- to sixday-old (block 1) and four- to seven-day-old adult mosquitoes (block 2) deprived of sucrose for 24 hours were offered an infectious blood meal for 30 min via membrane feeding as described above. Samples of the blood meal were saved for subsequent viral titration by fluorescent focus assay (FFA) in C6/36 cells [54]. Preliminary trials showed that there was no detectable decrease in blood meal titer over a period of $30 \mathrm{~min}$. After feeding, mosquitoes were briefly knocked-down with $\mathrm{CO}_{2}$ and fully engorged females were transferred to paper cups and held in a Precision incubator at $27 \pm 1^{\circ} \mathrm{C}$ and under $12: 12$ hour light:dark cycle and supplied with $10 \%$ sucrose ad libitum. High humidity in the incubator was maintained with containers filled with water.

\section{Vector competence}

The intrinsic ability of mosquitoes to transmit DENV was assessed 14 dpi with two standard phenotypes used in vector competence studies: (i) midgut infection and (ii) viral dissemination from the midgut to other tissues. Midgut infection was determined by detecting the presence of virus in mosquito bodies (thorax and abdomen). Viral dissemination was determined by detection of virus in mosquito legs and heads. Virus titers in the head and the legs of the same individual were strongly correlated among infected mosquitoes (linear regression: $\mathrm{R}^{2}=0.522$, $P<0.0001)$. We, therefore, used their average in the quantitative analyses of virus dissemination. We used both categorical (infection and dissemination status) and continuous measures (viral RNA concentration in bodies, virus titer in heads/legs) of vector competence components. We also recorded the proportion of dead mosquitoes at 14 dpi to account for any differential mortality between experimental groups. Mosquitoes were anesthetized with triethylamine (Sigma-Aldrich, St. Louis, MO), and their legs and heads were removed individually and transferred separately into $0.5 \mathrm{ml}$ of mosquito diluent (MD), consisting of $20 \%$ heat-inactivated fetal bovine serum (FBS) in Dulbecco's phosphate-buffered saline (PBS) with $50 \mu \mathrm{g} / \mathrm{ml}$ penicillin/streptomycin, $50 \mu \mathrm{g} / \mathrm{ml}$ gentamicin, and $2.5 \mu \mathrm{g} / \mathrm{ml}$ fungizone. The remainder of the mosquito bodies were placed individually into $0.7 \mathrm{ml}$ of $\mathrm{MD}$, and all samples were stored at $-80^{\circ} \mathrm{C}$ before processing. Samples were thawed on ice and homogenized in a mixer mill (Qiagen, Valencia, CA) at 24 cycles/ sec for 2 min. Infected bodies were screened by TaqMan ${ }^{\circledast}$ quantitative RT-PCR (qRT-PCR). Although viral RNA molecules are not equivalent to infectious virions, our conservative qRT-PCR detection threshold $\left(10^{4}\right.$ viral RNA copies per sample, see below) allowed us to consider that positive bodies contained $>1$ infectious virion because viral RNA copy numbers are 100- to 10,000-fold higher than virus titers $[21,55]$. Disseminated infections in the heads and legs of mosquitoes whose body was positive by qRT-PCR was determined by FFA in green monkey kidney cells (Vero cells, ATCC \#CCL-81) as described in [54]. Mosquitoes whose bodies were negative by qRT-PCR were 
considered uninfected and their legs and heads were not processed further. We verified the validity of the screening scheme by confirming that the bodies, legs, and heads of a random sub-sample of individuals negative by qRT-PCR were also negative by FFA.

\section{Quantitative RT-PCR}

Viral RNA was quantified in mosquito bodies by a serotype-specific, one-step TaqMan ${ }^{\circledR}$ qRT-PCR method modified from [56]. Briefly, RNA was extracted and purified using a semi-automated Prism 6100 Nucleic Acid Prepstation (Applied Biosystems, Foster City, CA). A sample of $300 \mu \mathrm{l}$ of each mosquito homogenate was pre-filtered and lysed with $300 \mu \mathrm{l}$ of $2 \times$ Nucleic Acid Purification Solution, and $500 \mu \mathrm{l}$ of the resulting lysate was deposited in a Purification Tray. Isolated RNA was washed once with $500 \mu \mathrm{l}$ of Wash Buffer 1, once with $500 \mu$ l of Wash Buffer 2, twice with $300 \mu \mathrm{l}$ of Wash Buffer 2, and finally eluted in $60 \mu \mathrm{l}$ of Elution Solution. qRT-PCR was carried out in 96-well plates with a 7900HT Fast Real-Time PCR system (Applied Biosystems) using a TaqMan ${ }^{\circledast}$ One-Step RT-PCR Master Mix Reagents kit (Applied Biosystems). Each sample was assayed in a $50 \mu \mathrm{l}$ reaction containing $10 \mu \mathrm{l}$ of RNA template, $0.5 \mu \mathrm{M}$ of forward primer, $1 \mu \mathrm{M}$ of reverse primer, and $0.25 \mu \mathrm{M}$ of fluorogenic DENV-1 specific probe. Forward and reverse primer sequences NS5F and NS5R reported in [56] were used to generate an amplicon of 104 bp within the NS5 gene. The dual-labeled DENV-1 specific probe sequence used in this study (5'-[6-FAM]-CTCAGAGACATATCAAAGATTCCAGC-[BHQ1]-3') was slightly modified from the DSQ1 sequence reported in [56] so that the target region of the NS5 gene was strictly identical among our three isolates (as confirmed by sequencing). The thermal profile consisted of an RT step at $48^{\circ} \mathrm{C}$ for 30 min, 10 min of Taq polymerase activation at $95^{\circ} \mathrm{C}$, followed by 40 cycles of PCR with $30 \mathrm{sec}$ of denaturation at $95^{\circ} \mathrm{C}$ and $1 \mathrm{~min}$ of annealing/extension at $60^{\circ} \mathrm{C}$. RNA solutions of known concentration were synthesized by in vitro transcription (IVT) and used as RNA standards for absolute quantification across plates [56]. A standard RTPCR was carried out on viral genomic RNA using a forward primer including a T7 promoter sequence. The T7 forward primer sequence was the IVT NS5F sequence reported in [56] and the reverse primer sequence was the cFD4 sequence reported in [57]. PCR products were cleaned up using the QIAquick PCR Purification kit (Qiagen) and $1 \mu \mathrm{g}$ of DNA was subjected to IVT using the T7 MAXIscript IVT kit (Ambion, Austin, TX) to generate a transcript of 480 bp containing the target NS5 region. IVT products were treated with TurboDNase (Ambion) at $37^{\circ} \mathrm{C}$ for $15 \mathrm{~min}$ and RNA was cleaned using the RNeasy MinElute kit (Qiagen). RNA was re-suspended in DEPCtreated water and molecular concentration was calculated by converting the optical density at $260 \mathrm{~nm}$ into the molecular copy number [56]. Solutions of concentrations ranging from $10^{7}$ to $10^{1} \mathrm{RNA}$ copies/ $\mu \mathrm{l}$ were aliquoted and stored at $-80^{\circ} \mathrm{C}$ until used to generate a standard curve for each TaqMan ${ }^{\circledast}$ plate. Baseline fluorescence was set automatically to account for differing starting RNA quantities between samples. The threshold level of fluorescence for threshold cycle (Ct) determination was optimized manually so that the slope of the standard curve was as close as possible to the theoretical value -3.32 which corresponds to $100 \%$ PCR efficiency. Positive and negative controls were included on each plate. For all plates the standard curve had an $\mathrm{R}^{2}>0.985$ and the detection limit was $10^{3}$ RNA copies $/ \mu$ l; i.e., $10^{4}$ RNA copies per $10 \mu$ l of RNA template.

\section{Fluorescent focus assay}

Virus titers were quantified using a tissue-culture assay based on immuno-fluorescent detection of infectious foci developing in cell monolayers [54]. Blood meal titers were assayed in C6/36 cells to provide a relevant estimate of viral infectivity to mosquito cells, whereas virus titers in mosquito heads and legs were assayed in Vero cells because we were interested in the eventual infectivity to a mammalian host. Cells were grown in $75-\mathrm{cm}^{2}$ flasks in Dulbecco's Modified Eagle Medium with 4,500 mg/l of Dglucose and L-glutamine (DMEM, Invitrogen) supplemented with $10 \%$ FBS, $1.5 \mathrm{~g} / \mathrm{l}$ sodium bicarbonate, 100 $\mathrm{U} / \mathrm{ml}$ penicillin, and $100 \mu \mathrm{g} / \mathrm{ml}$ streptomycin. Eight-well chambered slides were seeded with Vero or C6/36 cells at a density of $2.5 \times 10^{5}$ or $5.0 \times 10^{6}$ cells/well, respectively, and incubated for 24 hours at $37^{\circ} \mathrm{C}$ (Vero) or $28^{\circ} \mathrm{C}(\mathrm{C} 6 /$ 36 ) under $5 \% \mathrm{CO}_{2}$, to produce a confluent monolayer. Ten-fold serial dilutions of blood meal samples or head/ legs homogenates were inoculated onto cell monolayers in a final volume of $50 \mu \mathrm{l} /$ well. Viral adsorption was allowed to proceed for one hour at $37^{\circ} \mathrm{C}$ (Vero) or $28^{\circ} \mathrm{C}$ $(\mathrm{C} 6 / 36)$ under $5 \% \mathrm{CO}_{2}$, rocking the slides every $15 \mathrm{~min}$. At the conclusion of the adsorption, an overlay of growth medium with $5 \%$ FBS, and $0.8 \%$ carboxymethylcellulose (CMC, Sigma-Aldrich) was added in a final volume of 0.5 $\mathrm{ml} /$ well. DENV infectious foci develop faster in Vero than in C6/36 cells [54]. After an incubation of 48 hours at $37^{\circ} \mathrm{C}$ (Vero) or 72 hours at $28^{\circ} \mathrm{C}(\mathrm{C} 6 / 36)$ under $5 \% \mathrm{CO}_{2}$, the overlay medium was removed from the infected monolayers and the cells were washed twice with cold PBS. Infected monolayers were fixed for $10 \mathrm{~min}$ in ice-cold absolute methanol (Sigma-Aldrich) and washed once with PBS. Slides were incubated for one hour with a mouse anti-dengue complex primary antibody clone D32H2-9-21 (Millipore, Temecula, CA) diluted 1:200 in PBS containing $0.2 \%$ bovine serum albumin (PBS-BSA $0.2 \%$ ). After three washes in PBS-BSA 0.2\%, slides were incubated for $30 \mathrm{~min}$ with a goat anti-mouse fluorescein-conjugated secondary antibody (Millipore) diluted 1:50 in PBS-BSA $0.2 \%$, followed by three washes in PBS-BSA $0.2 \%$ and a final wash in distilled water. Cells were mounted in 
Vectashield anti-fading medium (Vector Laboratories, Burlingame, CA) and observed under a $20 \times$ objective on an Olympus Provis fluorescence microscope equipped with a FITC filter. The total number of fluorescent foci in each well was visually counted and virus titers were calculated as fluorescent focus units (FFUs) per ml. Reading was done blindly by number-coding and randomizing samples on the slides.

\section{Data analysis}

We first analyzed differences in body size between isofemale families by comparing wing lengths using a one-way analysis of variance (ANOVA). Then, because our experiment fulfilled the characteristics of a randomized complete block design [58], we used full-factorial analyses including the factors mosquito family, virus isolate, experimental block, and all their interactions. Categorical variables (infection and dissemination status) were analyzed with multi-way logistic nominal regressions, whereas continuous variables (viral RNA concentration in bodies, virus titer in heads/legs, mortality) were analyzed with multi-way ANOVAs. In order to satisfy the assumptions of the statistical tests (in particular, normality of the residuals), we used a logarithmic transformation of viral RNA concentrations and virus titers and an arcsine-transformation of mortalities. The proportion of mosquitoes with a disseminated infection and concentration of viral RNA in bodies were analyzed among infected mosquitoes (i.e., excluding uninfected mosquitoes). The average virus titer of heads and legs was analyzed among mosquitoes with a disseminated infection (i.e., excluding mosquitoes that did not have a disseminated infection). Differences were considered statistically significant at $P<0.05$. All statistical analyses were performed with the software JMP version 5.1.2 http://www.jmpdiscovery.com.

\section{Authors' contributions}

LL conceived the study, performed the research, analyzed the data, and wrote the manuscript. CC helped to design the study and draft the manuscript. RGA participated in the vector competence assays. BT carried out the isolation and amplification of the viruses. JHR coordinated the field collection of mosquitoes. RGJ coordinated the collection of virus isolates. TWS participated in the design and coordination of the study, and helped to draft the manuscript. All authors read and approved the final manuscript. The opinions or assertions contained herein are the private views of the authors and are not to be construed as reflecting the official views of the United States Army, Royal Thai Army, or the United States Department of Defense.

\section{Acknowledgements}

The authors thank Jittawadee Murphy, Prasan Kankaew, Somporn Changimongkol, Thanyalak Fansiri, Siriporn Phasomkusolsil, Yossasin Kertmanee, Kanchana Pantuwatana, and Jaruwan Tawong for technical assistance during mosquito collections. We are grateful to William Reisen and Aaron Brault for their continuous support through access to the Center for Vectorborne Disease Research. Thanks to Ying Fang, Sandy Garcia, Keira Simmons, Stanley Langevin and Rajeev Vaidyanathan for their help with cell culture and virological assays, and to two anonymous reviewers for valuable comments on an earlier version of the manuscript. LL is supported by a post-doctoral Marie Curie Outgoing International Fellowship from the $6^{\text {th }}$ Framework Program of the European Commission. This work was partially funded by the French CNRS program 'Maladies Infectieuses Emergentes'.

\section{References}

I. Holmes EC, Burch SS: The causes and consequences of genetic variation in dengue virus. Trends Microbiol 2000, 8(2):74-77.

2. Rico-Hesse R: Microevolution and virulence of dengue viruses. Adv Virus Res 2003, 59:315-34I.

3. Zanotto PM, Gould EA, Gao GF, Harvey PH, Holmes EC: Population dynamics of flaviviruses revealed by molecular phylogenies. Proc Natl Acad Sci USA 1996, 93(2):548-553.

4. Gubler DJ: Dengue and dengue hemorrhagic fever. Clin Microbiol Rev 1998, I I (3):480-496.

5. Guzman MG, Kouri G: Dengue: an update. Lancet Infect Dis 2002, 2(I):33-42.

6. Gubler DJ: Epidemic dengue/dengue hemorrhagic fever as a public health, social and economic problem in the 2 Ist century. Trends Microbiol 2002, I0(2):100-103.

7. Holmes EC, Twiddy SS: The origin, emergence and evolutionary genetics of dengue virus. Infect Genet Evol 2003, 3(I):19-28.

8. Jarman RG, Holmes EC, Rodpradit $P$, Klungthong $C$, Gibbons RV, Nisalak A, Rothman AL, Libraty DH, Ennis FA, Mammen MP Jr, et al.: Microevolution of Dengue viruses circulating among primary school children in Kamphaeng Phet, Thailand. J Virol 2008, 82(I I):5494-5500.

9. Chevillon $C$, Failloux $A B$ : Questions on viral population biology to complete dengue puzzle. Trends Microbiol 2003, I I(9):4|5-42I.

10. Diaz FJ, Black WC 4th, Farfan-Ale JA, Lorono-Pino MA, Olson KE, Beaty BJ: Dengue virus circulation and evolution in Mexico: a phylogenetic perspective. Arch Med Res 2006, 37(6):760-773.

II. Myat Thu H, Lowry K, Jiang L, Hlaing T, Holmes EC, Aaskov J: Lineage extinction and replacement in dengue type I virus populations are due to stochastic events rather than to natural selection. Virology 2005, 336(2): 163-172.

12. Bennett SN, Holmes EC, Chirivella M, Rodriguez DM, Beltran M, Vorndam V, Gubler DJ, McMillan WO: Selection-driven evolution of emergent dengue virus. Mol Biol Evol 2003, 20(10): I 650-1658.

13. Bennett SN, Holmes EC, Chirivella M, Rodriguez DM, Beltran M, Vorndam V, Gubler DJ, McMillan WO: Molecular evolution of dengue 2 virus in Puerto Rico: positive selection in the viral envelope accompanies clade reintroduction. J Gen Virol 2006, 87(Pt 4):885-893.

14. Twiddy SS, Farrar JJ, Vinh Chau N, Wills B, Gould EA, Gritsun T, Lloyd G, Holmes EC: Phylogenetic relationships and differential selection pressures among genotypes of dengue-2 virus. Virology 2002, 298(I):63-72.

15. Twiddy SS, Woelk CH, Holmes EC: Phylogenetic evidence for adaptive evolution of dengue viruses in nature. J Gen Virol 2002, 83(Pt 7): 1679-1689.

16. Zhang C, Mammen MP Jr, Chinnawirotpisan P, Klungthong C, Rodpradit P, Nisalak A, Vaughn DW, Nimmannitya S, Kalayanarooj S, Holmes EC: Structure and age of genetic diversity of dengue virus type 2 in Thailand. J Gen Virol 2006, 87(Pt 4):873-883.

17. Tsetsarkin KA, Vanlandingham DL, McGee CE, Higgs S: A Single Mutation in Chikungunya Virus Affects Vector Specificity and Epidemic Potential. PLoS Pathog 2007, 3( I 2):e20I.

18. Vazeille M, Moutailler S, Coudrier D, Rousseaux C, Khun H, Huerre M, Thiria J, Dehecq JS, Fontenille D, Schuffenecker I, et al.: Two Chikungunya isolates from the outbreak of La Reunion (Indian Ocean) exhibit different patterns of infection in the mosquito, Aedes albopictus. PLoS ONE 2007, 2(I I):el I 68.

19. Moudy RM, Meola MA, Morin LL, Ebel GD, Kramer LD: A newly emergent genotype of West Nile virus is transmitted earlier and more efficiently by Culex mosquitoes. Am J Trop Med Hyg 2007, 77(2):365-370. 
20. Anderson JR, Rico-Hesse R: Aedes aegypti vectorial capacity is determined by the infecting genotype of dengue virus. Am Trop Med Hyg 2006, 75(5):886-892.

21. Armstrong PM, Rico-Hesse R: Differential susceptibility of Aedes aegypti to infection by the American and Southeast Asian genotypes of dengue type 2 virus. Vector Borne Zoonotic Dis 200I, I(2): I59-I68.

22. Armstrong PM, Rico-Hesse R: Efficiency of dengue serotype 2 virus strains to infect and disseminate in Aedes aegypti. Am J Trop Med Hyg 2003, 68(5):539-544

23. Hanley KA, Nelson JT, Schirtzinger EE, Whitehead SS, Hanson CT: Superior infectivity for mosquito vectors contributes to competitive displacement among strains of dengue virus. BMC Ecol 2008, 8: I.

24. Apostol BL, Black WC 4th, Reiter P, Miller BR: Population genetics with RAPD-PCR markers: the breeding structure of Aedes aegypti in Puerto Rico. Heredity 1996, 76(Pt 4):325-334

25. Bosio CF, Harrington LC, Jones JW, Sithiprasasna R, Norris DE, Scott TW: Genetic structure of Aedes aegypti populations in Thailand using mitochondrial DNA. Am J Trop Med Hyg 2005, 72(4):434-442.

26. Garcia-Franco F, de Lourdes Munoz M, Lozano-Fuentes S, FernandezSalas I, Garcia-Rejon J, Beaty BJ, Black WC 4th: Large genetic distances among Aedes aegypti populations along the South Pacific coast of Mexico. Am J Trop Med Hyg 2002, 66(5):594-598.

27. Huber K, Le Loan L, Hoang TH, Ravel S, Rodhain F, Failloux AB: Genetic differentiation of the dengue vector, Aedes aegypti (Ho Chi Minh City, Vietnam) using microsatellite markers. Mol Ecol 2002, I I(9): I629-I635.

28. Black WC 4th, Bennett KE, Gorrochotegui-Escalante N, Barillas-Mury CV, Fernandez-Salas I, de Lourdes Munoz M, Farfan-Ale JA, Olson KE, Beaty BJ: Flavivirus susceptibility in Aedes aegypti. Arch Med Res 2002, 33(4):379-388.

29. Greischar MA, Koskella B: A synthesis of experimental work on parasite local adaptation. Ecol Lett 2007, I0(5):4 I8-434.

30. Joy DA, Gonzalez-Ceron L, Carlton JM, Gueye A, Fay M, McCutchan TF, Su XZ: Local adaptation and vector-mediated population structure in Plasmodium vivax malaria. Mol Biol Evol 2008 , 25(6): $1245-1252$

31. Gandon S: Local adaptation and host-parasite interactions. Trends Ecol Evol 1998, I3(6):214-216.

32. Kaltz O, Shykoff JA: Local adaptation in host-parasite systems. Heredity 1998, 8 I:36I-370.

33. Kawecki TJ, Ebert D: Conceptual issues in local adaptation. Ecol Lett 2004, 7( I 2): | 225-|24|.

34. Lambrechts L, Fellous S, Koella JC: Coevolutionary interactions between host and parasite genotypes. Trends Parasitol 2006 , 22(I): $12-16$

35. Bennett KE, Olson KE, de Lourdes Munoz M, Fernandez-Salas I, Farfan-Ale JA, Higgs S, Black WC 4th, Beaty BJ: Variation in vector competence for dengue 2 virus among 24 collections of Aedes aegypti from Mexico and the United States. Am J Trop Med Hyg 2002, 67(I):85-92.

36. Gubler DJ, Nalim S, Tan R, Saipan H, Sulianti Saroso J: Variation in susceptibility to oral infection with dengue viruses among geographic strains of Aedes aegypti. Am J Trop Med Hyg 1979, 28(6): $1045-1052$.

37. Tardieux I, Poupel O, Lapchin L, Rodhain F: Variation among strains of Aedes aegypti in susceptibility to oral infection with dengue virus type 2. Am J Trop Med Hyg 1990, 43(3):308-3।3.

38. Vazeille-Falcoz M, Mousson L, Rodhain F, Chungue E, Failloux AB Variation in oral susceptibility to dengue type 2 virus of populations of Aedes aegypti from the islands of Tahiti and Moorea, French Polynesia. Am J Trop Med Hyg 1999, 60(2):292-299.

39. Mousson L, Vazeille M, Chawprom S, Prajakwong S, Rodhain F, Failloux $A B$ : Genetic structure of Aedes aegypti populations in Chiang Mai (Thailand) and relation with dengue transmission. Trop Med Int Health 2002, 7(1 0):865-872.

40. Tran TK, Vazeille-Falcoz M, Mousson L, Hoang TH, Rodhain F, Huong NT, Failloux AB: Aedes aegypti in Ho Chi Minh City (Viet Nam): susceptibility to dengue 2 virus and genetic differentiation. Trans Roy Soc Trop Med Hyg 1999, 93(6):581-586.

4I. Rosen L, Roseboom LE, Gubler DJ, Lien JC, Chaniotis BN: Comparative susceptibility of mosquito species and strains to ora and parenteral infection with dengue and Japanese encephalitis viruses. Am J Trop Med Hyg I985, 34(3):603-6I5.

42. Beerntsen BT, James AA, Christensen BM: Genetics of mosquito vector competence. Microbiol Mol Biol Rev 2000, 64(I): I I5-I 37.

43. Alto BW, Reiskind MH, Lounibos LP: Size alters susceptibility of vectors to dengue virus infection and dissemination. Am Trop Med Hyg 2008, 79(5):688-695.

44. Lynch M, Walsh B: Genetics and Analysis of Quantitative Traits. Sunderland, Massachusetts: Sinauer Associates; 1998.

45. Wilfert $L$, Schmid-Hempel $P$ : The genetic architecture of susceptibility to parasites. BMC Evol Biol 2008, 8: 187

46. Gomez-Machorro C, Bennett KE, de Lourdes Munoz M, Black WC 4th: Quantitative trait loci affecting dengue midgut infection barriers in an advanced intercross line of Aedes aegypti. Insect Mol Biol 2004, I 3(6):637-648.

47. Bennett KE, Flick D, Fleming KH, Jochim R, Beaty BJ, Black WC 4th: Quantitative trait loci that control dengue-2 virus dissemination in the mosquito Aedes aegypti. Genetics 2005, I 70(I): I85-194.

48. Bosio CF, Fulton RE, Salasek ML, Beaty BJ, Black WC 4th: Quantitative trait loci that control vector competence for dengue-2 virus in the mosquito Aedes aegypti. Genetics 2000 , I 56(2):687-698.

49. Aaskov J, Buzacott K, Thu HM, Lowry K, Holmes EC: Long-term transmission of defective RNA viruses in humans and Aedes mosquitoes. Science 2006, 3 I I (5758):236-238.

50. Lin SR, Hsieh SC, Yueh YY, Lin TH, Chao DY, Chen WJ, King CC, Wang WK: Study of sequence variation of dengue type 3 virus in naturally infected mosquitoes and human hosts: implications for transmission and evolution. J Virol 2004, 78(22): |27|7-|272|

5I. Grech K, Maung LA, Read AF: The effect of parental rearing conditions on offspring life history in Anopheles stephensi. Malar | 2007, 6: 130 .

52. Niaré O, Markianos K, Volz J, Oduol F, Touré A, Bagayoko M, Sangaré $D$, Traoré SF, Wang R, Blass C, et al.: Genetic loci affecting resistance to human malaria parasites in a West African mosquito vector population. Science 2002, 298(5591):213-216.

53. Craig GB Jr: Mosquitoes: female monogamy induced by male accessory gland substance. Science 1967, I56(78I):|499-I50I.

54. Payne AF, Binduga-Gajewska I, Kauffman EB, Kramer LD: Quantitation of flaviviruses by fluorescent focus assay. J Virol Methods 2006, I 34(I-2): 183-189.

55. Richardson J, Molina-Cruz A, Salazar MI, Black W 4th: Quantitative analysis of dengue-2 virus RNA during the extrinsic incubation period in individual Aedes aegypti. Am J Trop Med Hyg 2006, 74(I): |32-|4|.

56. Kong $\mathrm{YY}$, Thay $\mathrm{CH}$, Tin TC, Devi S: Rapid detection, serotyping and quantitation of dengue viruses by TaqMan real-time one-step RT-PCR. J Virol Methods 2006, I38(I-2): I23-130.

57. Kuno G, Chang G], Tsuchiya KR, Karabatsos N, Cropp CB: Phylogeny of the genus Flavivirus. J Virol I998, 72(I):73-83

58. Sokal RR, Rohlf FJ: Biometry: the principles and practice of statistics in biological research. 3rd edition. New York: W. H. Freeman and Co; 1995.

Publish with Biomed Central and every scientist can read your work free of charge

"BioMed Central will be the most significant development for disseminating the results of biomedical research in our lifetime. "

Sir Paul Nurse, Cancer Research UK

Your research papers will be:

- available free of charge to the entire biomedical community

- peer reviewed and published immediately upon acceptance

- cited in PubMed and archived on PubMed Centra

- yours - you keep the copyright 